\title{
Adipose derived stem cells (ADSCs) Immunomodulation Impact on Skin Tissue Repair
}

\section{Mazini $\mathrm{L}^{1 *}$, Rochette $\mathrm{L}^{2}$, Amal $\mathrm{S}^{3}$, Admou $\mathrm{B}^{4}$ and Malka $\mathrm{G}^{1}$}

${ }^{1}$ Stem Cells and Cellular and Tissue Regeneration Laboratory, Medical Applications Interface Center (CIAM), Mohammed VI Polytechnic University, Ben-Guerir, Morocco

${ }^{2}$ Host Team (EA 7460), Cerebro-Cardiovascular Physiopathology and Epidemiology (PEC2), University of Burgundy-Franche Comte, Dijon, France

${ }^{3}$ Department of Dermatology, University Hospital Center, Marrakech, Morocco

${ }^{4}$ Immunology laboratory, Clinical Research Center, Faculty of Medicine and Pharmacy, Cadi Ayyad University, Morocco

\section{Review Article}

Volume 4 Issue 1

Received Date: February 06, 2020

Published Date: February 14, 2020

DOI: $10.23880 /$ jes-16000136

*Corresponding author: Loubna Mazini, Stem Cells and Cellular and Tissue Regeneration Laboratory, Medical Applications Interface Center (CIAM), Mohammed VI Polytechnic University, 43150 Ben-Guerir, Morocco, Tel: +212666392899; Email: loubna.mazini@um6p.ma

\section{Abstract}

Adipose derived stem cells (ADSCs) have largely proven their efficiency in wound healing and skin regeneration in vitro and in vivo. They are niched within the hypodermis where they differentiate into skin cell types and migrate to wounded sites to restore cell deficiencies and functions. ADSCs mainly act by an autocrine pathway through their secretome containing growth factors, cytokines and microRNA (miR) involved in their migration and proliferation and that of dermal fibroblasts (DF) and keratinocytes. There are evidences arguing that the immunomodulation capacity of ADSCs reflect their efficacy to ensure skin tissue repair. Their secretome is implicated in immune responses and is considered as the key tool in regulating skin inflammation and diseases-associated inflammation. By their action on skin immune cells, ADSCs are expected to play the principal role by modulating: $i$ : the expression of the inflammatory factors; ii: the activation and maturation of immune cells especially M1 and M2 macrophages and inhibition of Th cells; iii: the skin cell differentiation, proliferation, pigmentation and migration and iv: the secretion of the extracellular matrix (ECM) proteins. Additionally, ADSCs cross-react with the different skin cells, ECM and the surrounding growth factors to initiate and accelerate the implementation of all phases of wound healing. In this review, we highlight the role of ADSCs in deriving tissue repair with regard to their immune-modulation ability, this impressive capacity should be considered when using ADSCs in regenerative medicine.

Keywords: Adipose Derived Stem Cells; Skin; Immunomodulation; Regeneration; Repair; Inflammation; Wound Healing

Abbreviations: ADSCs: Adipose Derived Stem Cells; GDF11: Growth Differentiation Factor; TGF- $\beta$ : Transforming Growth Factor; ECM: Extracellular Matrix; DF: Dermal Fibroblasts; MSCs: Mesenchymal Stem Cells; IL-1, -6, -8, -10:
Interleukin-1, -6, -8, -10; TNF- $\alpha$ : Tumor Necrosis Factor- $\alpha$; VEGF: Vascular Endothelial Growth Factor; PDGF: Platelets Derived Growth Factor; MCP-1: Monocyte Chemoattractant Protein-1; b-FGF: Basic-Fibroblast Growth Factor; TLR2, 


\section{Journal of Embryology \& Stem Cell Research}

4: Toll-Like Receptor2,4; GM-CSF: Granulocyte MonocyteColony Stimulating Factor; G-CSF: Granulocyte-Colony Stimulating Factor; IGF: Insulin Growth Factor; HGF: Hepatocyte Growth Factor.

\section{Introduction}

Adipose derived stem cell (ADSC)-based therapy is very promising in treating damaged tissues and in completing fullthickness skin replacement. These cells are identified within the subcutaneous adipose tissue and have been reported to differentiate into skin cells ensuring thus skin regeneration and maintaining homeostasis [1-3]. Clinical applications benefit from its simple and abundant collection from adipose tissue. The capacity of these cells to proliferate and self-renew in vitro as well as in vitro, added to their innate differentiation targeted more scientific advancements in the field of regenerative medicine $[4,5]$. Several studies have shown the ability of ADSC to act through cell-cell contact, but mostly by secreting a panel of cytokines and chemokines, being involved in different biological pathways including cell proliferation, differentiation, homing and migration, senescence and apoptosis [6-14]. These mechanisms are implicated in the whole process of skin regeneration and wound healing.

Their immunomodulatory effects make them more suitable for use compared to their counterpart from bone marrow and umbilical blood [15]. These cells have been used for many investigations and are largely used for graft improvement in cosmetic remodeling to prevent fat necrosis [16-18]. ADSCs present great ability in migration leading to their rapid recruitment into wounded sites where process of cell differentiation towards various skin cell components occurred. ADSCs are reported to regulate inflammation and participate to the phases of wound healing [3]. These cells gain more interest with their enriched extracellular vesicles containing growth factors, cytokines, MicroRNA (miR) and produce different amounts of the extracellular matrix (ECM) proteins thus promoting and accelerating skin regeneration [12-14,19-23].

This suggested that these cells represent a rich source of factors and are able to address their microenvironment to modulate wound healing and tissue regeneration.

Among the secreted growth factors, transforming growth factors- $\beta$ (TGF- $\beta$ ), growth differentiation factor 11 (GDF11), interleukin-6 (IL-6), IL-1 $\beta$, IL-8, IL-10, Toll like Receptor 2 (TLR2), TLR4 and TNF- $\alpha$ are highlighted and both involved in skin and different organs biology [6,11,2427]. These factors target similar skin cells including dermal fibroblast (DF), keratinocytes, melanocytes and dermal micro vascular endothelial cells [28-30]. Additionally, ADSCs secrete TGF- $\beta$ leading to activation of macrophages in combination to IL- 6 and IL-1 $\beta$ [31].

Therefore, ADSCs can be differentiated towards a desired cell phenotype as a result of microenvironment stimulation or by an appropriate composition of cell culture media. Indeed, ADSC's functions are modulated by the composition and the presence of immune cells of their microenvironment and change their behavior in response to the nature of biomolecules, cytokines or cellular profile present [3236]. Their conditioned media has the ability to induce macrophages to secrete IL-10 and TNF anti-inflammatory cytokines, and at the other side to decrease the secretion of the pro- inflammatory cytokines $[28,37]$.

Also, aging is another parameter impacting the immunomodulation effect of ADSCs. Accumulation of senescent cells result on an increase in the pro-inflammatory factors as IL-6, IL-8 and TNF- $\alpha$ associated to chronic inflammation $[24,38]$.

We can consider that ADSCs are likely the main actor involved in skin regeneration, their ability to self-renew, to differentiate into skin cells, to modulate the duration of the inflammatory phase and the re-epithelialization is potentially dependent on the enrichment of their secretome and their cellular network within skin layers. More investigations are however needed to better address their relative relationships within their microenvironment.

\section{ADSCs Interactions in Tissue Repair}

Increasing evidences implicated ADSCs in maintaining skin homeostasis at a cellular level through cell differentiation and at the paracrine level. During normal development, skin regenerative capability is performed by the resident ADSCs providing for cellular turn over during skin homeostasis and repair after injury [33]. Basal layer is the skin location where these active multipotent stem cells are responsible for recruiting and sending mature differentiated cells (keratinocytes) to the outer of epidermis. Through a hierarchic gradient, these stem cells induced epidermis layer regeneration by ensuring self-renewal and a continuously production of transient amplifying cells [39]. Epidermal cells including ADSCs and DF are closely interacted to maintain local microenvironment propitious for cell turnover leading to skin regeneration. Adding to their aptitude to differentiate into keratinocytes, DF and probably melanocytes, crosstalk of ADSCs and these cells is a part of normal skin function where ECM secretion leaded to a physical environment critical for the maintenance of the stem cell niche [40].

Moreover, ADSCs secrete exosomes to modulate the homeostasis of skin microenvironment by releasing 


\section{Journal of Embryology \& Stem Cell Research}

the growth factors involving in neo-angiogenesis, cell differentiation, cell proliferation and in migration $[11,19,41$ 43].

Another proof of evidence of ADSCs interactions within skin cell and especially DF has been provided by $\mathrm{Hu}$ et al where skin fibroblasts cell line HS27 was found to activate ADSCs to differentiate into fibroblast-like cells highly expressing vimentin, HSP47 and desmin mRNA level [44]. The interactions of microvascular endothelial cells and ADSCs are also of great interest in skin cell regeneration and proliferation by providing IL-6, IL-8, monocyte chemoattractant protein-1 (MCP-1) and VEGF leading to inflammation and angiogenesis regulation $[45,46]$.

\section{The Secretome of ADSCs}

In normal conditions, ADSCs are continuously activated by human serum and platelets to induce their proliferation and differentiation. Once the tissues are wounded, platelets induced stem cells to initiate the inflammatory phase by secreting platelet- derived growth factor (PDGF), IL-6 and IL-8 which lead to migration of macrophages and neutrophils to the wounded site [47], and TGF- $\beta$ inducing induction of monocytes to macrophages. With similarity to platelets, ADSCs additionally secrete, prostaglandin E2 (PGE2), TNF- $\alpha$ and GDF11 potentiating thus the proinflammatory responses and later anti-inflammatory cytokines secretion by polarizing macrophages from M1 to M2.

In addition to TGF- $\beta$ and GDF11, ADSCs secrete other growth factors such as basic- fibroblast Growth Factor (b-FGF), stromal-derived factor-1 (SDF-1), insulin-like growth factor (IGF), hepatocyte growth factor (HGF) and wingless 10b (Wnt10b), IL6, Il-10, IL-1, TLR2, TLR4, G-CSF, GM-CSF and many others. These factors are included in the mechanisms regulating skin cell regeneration and repair $[6,7,28]$. The factors VEGF, PDGF, TGF- $\beta, b-F G F$ and HGF promoted new blood vessels during the proliferative phase, probably through their differentiation into endothelial progenitor cells $[6,48]$. The secreted GM-CSF can take part of this activation by inducing differentiation into committed monocytes potentially activated to macrophages and endothelial cells.

Actually, other cytokines and miR are expected to play a key role in skin tissue regeneration such as PDGF, IL-1, Bone Morphogenic Protein (BMP) 6, BMP9, miR-21, miR-23A and miR-124 by targeting DF, keratinocytes and especially immune cells $[12,14]$.

ADSCs are expected to participate actively to ECM production, whereby its abundant accumulation would facilitate cell migration and angiogenesis by auto induction and amplifying the growth factor secretion implied. However, endothelial cells and macrophages migration occurred probably once after macrophages leaded to secretion of the ECM proteins especially collagen I and III, elastin and fibronectin and to activation of DF to proliferate and migrate. DF also secrete VEGF, TGF- $\beta$ and $b$-FGF leading to angiogenesis $[48,49]$.

The collagen production amplified by cross-talking between ADSCs, DF and TGF- $\beta$ would facilitate remodeling phase through inhibiting ECM degradation by increasing TIMPs secretion and their binding to MMPs [50].Xiao, et al. have reported that adipose tissue secretome increased $\mathrm{N}$-cadherin and CD44 adhesion molecules involved in fibroblasts motility during wound healing and stimulating fibronectin expression during ECM remodeling [51]. Combination of activin B and ADSCs leaded to rapid wound closure and to accelerating epithelialization through promoting keratinocytes and fibroblasts proliferation [2]. The $\alpha \beta 6$ integrin exclusively expressed by epithelial cells, was associated to the regeneration of basement membrane zone during wound repair [52]. When miming injured conditions in vitro, ADSCs were indeed demonstrated to accelerate neovascularization through the expression of hypoxia- inducible factor-1A [53] by regulating VEGF gene expression in endothelial cells [54].

\section{Immunoregulatory Effects of ADSCs in Skin}

Evidences of involvement of ADSCs in immunomodulation of tissues and their presence within the epidermal layer have suggested that these cells might play a crucial role in skin immunological functions in physiologic and injured skin. Accumulation of senescent cells in the skin is related to the production of pro-inflammatory factors such as IL-6, IL-8, TNF- $\alpha$ thus changing the inflammatory profile of the associated microenvironment as in aged tissues [55]. This skin inflamm-aging was also supported by highly secreted pro-inflammatory cytokine IL-1 $\beta[24,38]$. Microvascular endothelial cells while interacting with ADSCs lead to increase secretion of IL-6, IL-8, MCP-1 modulating skin inflammation [46].

Migration of ADSCs to the injured site is of a pivotal interest, their immune profile and their potential shift towards a more anti-inflammatory phenotype is required for the proliferation and remodeling phases of healing [56-58]. The cytokine profile of T, B and dendritic cells was influenced by ADSCs which lead to the interruption of the inflammatory phase and initiate the proliferation and remodeling phases in chronic wounds $[59,60]$.

Preconditioning with inflammatory or pro-inflammatory cytokines has improved ADSC's responses to cancer and inflammation in addition to the increase of their survival 


\section{Journal of Embryology \& Stem Cell Research}

[61-64]. Several reports are in favor of optimizing the therapeutic benefit of ADSCs as a result of the combined action of cytokines including TGF- $\beta$ and TNF- $\alpha$. The potential implication of GDF11 in disrupting the pro-inflammatory status towards the anti-inflammatory one is likely due to its highly stimulation of DF and ADSCs and by amplifying the action of TGF- $\beta$ on ADSCs proliferation and secretome. This epigenetically modification and regulation of ADSC's microenvironment might be crucial in restoring cellular age defects and or increasing cell ability to differentiate and migrate.

TFG- $\beta$, secreted by ADSCs and other epithelial cells is involved in the inflammatory, proliferative and remodeling phases of wound healing [32]. Together with IL-1 $\beta$ and IL-6, this factor increases macrophages recruitment, and their polarization from M1 to M2 [31] leading to a phase of secretion of anti-inflammatory cytokines [65,66]. This activation helps the secretion of ECM proteins involved in skin structure repair, angiogenesis, cell proliferation and migration and re-epithelialization (Figure 1). The $\alpha \beta 6$ integrin secreted by epithelial cells was reported to activate TGF- $\beta$ modulating thus the innate immune surveillance in skin and ADSC microenvironment [52]. Recently, the collagen triple helix repeat containing 1 protein contributed to healing process via increasing M2 macrophages recruitment and TGF- $\beta$ expression level [67].

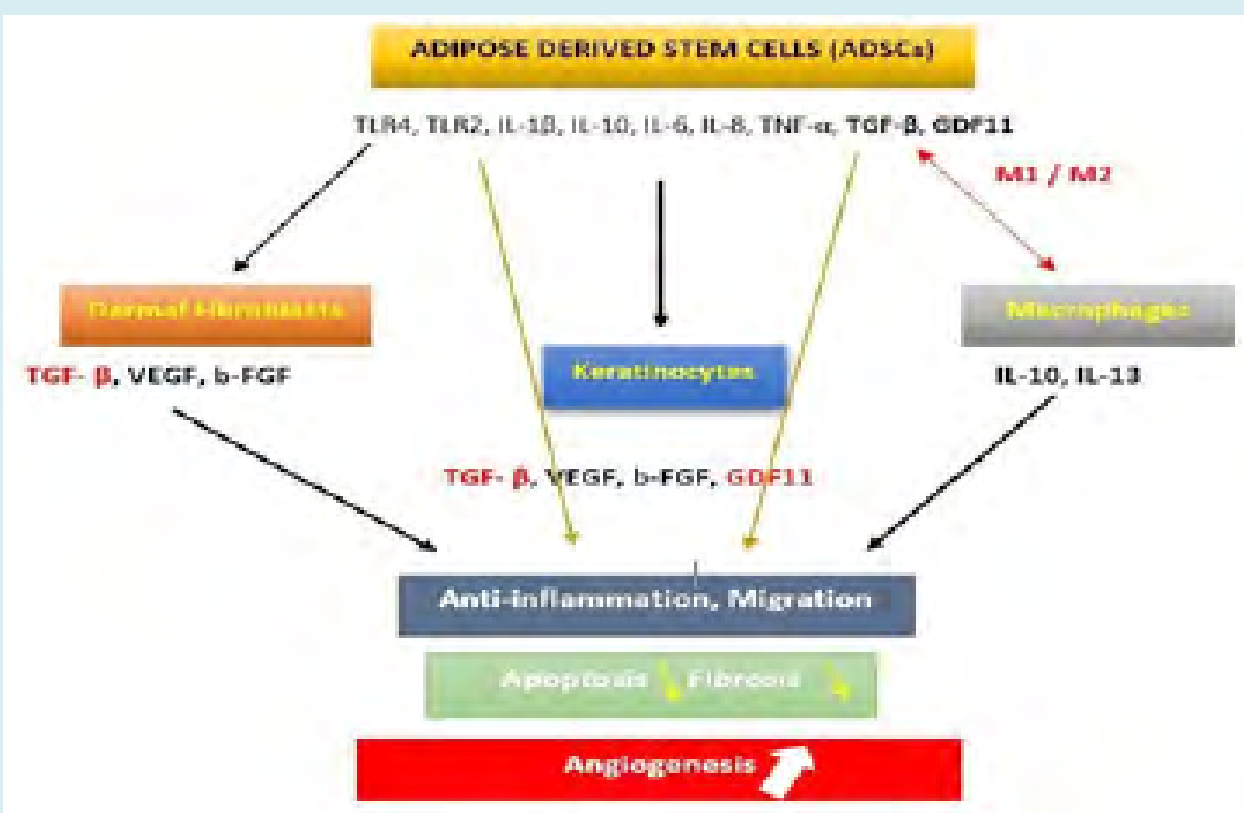

Figure 1: Immunomodulatory effects of ADSCs. Through their secretome, these cells change the macrophages polarization and acquire an anti-inflammatory profile to enhance skin cell proliferation and migration, improve angiogenesis accelerating thus re-epithelialization.

A recent work of Wang, et al. [68] has shown that skin treatment with rGDF11 decreased secretion of inflammatory cytokines by immune cells in vitro and in vivo in mice models of psoriasis-associated inflammation, an extended infiltration of immune cells sustaining skin inflammation. Suppression of severity of this inflammation by GDF11 was provided by reducing macrophages infiltration to the skin and inhibiting the NF- kB signaling pathway. Moreover, macrophages activation has been reported in skin autoimmune inflammatory diseases [69]. Other evidences argued on the protective effect of GDF11 from inflammatory reaction by inhibiting inflammatory responses in RAW264.7 macrophages probably by inhibiting TNF- $\alpha$ release $[70,71]$.
Additionally, TNF- $\alpha$ was activated by NF-kB signaling pathway during inflammation reaction being suppressed by GDF11 in atherosclerosis.

The impact of microenvironment changing on ADSCs functionality, on their induction of proliferation, differentiation and migration has already been reported $[32-35,72]$. Reciprocally, ADSCs are reported to address this microenvironment in vitro, their conditioned media was reported to activate macrophages and to increase the secretion of TNF and IL-10 anti-inflammatory cytokines stimulating thus wound healing $[37,73]$. 


\section{Journal of Embryology \& Stem Cell Research}

Other results have demonstrated the suppression of T-cell proliferation and TNF- $\alpha$ secretion when ADSCs were preconditioned with inflammatory media simultaneously to the significant upregulation of the anti-inflammatory genes expression and the IL-1 receptor antagonist and IL10 [73]. Another interesting in vitro study has reported that macrophages cultured with ADSCs have also the ability to inhibit the Тн 2 and TH17 cells and to increase significantly the frequency of Treg cells as the same time as adopting the healing and anti- inflammatory profile [74]. Using a specific mussel- inspired nanostructure scaffold derived ADSCs to shift macrophages from M1 to M2 immunomodulatory profile, to enhance vascularization thus accelerating wound closure [64]. This result also suggests the crosstalk between ADSCs and local immune cells to address the inflammatory status of their microenvironment which appear to be potentiated by direct cell-cell contact $[32,75]$.

At the same way, IL-6 the major pro-inflammatory components of ADSC's secretome was reported to initiate the inflammatory phase in injured tissues (Figure 1). Autoactivated ADSCs amplify their secretion and stimulate the secretion of TNF- $\alpha$, TLR2, TLR4, IL-10, b-FGF, VEGF, TGF- $\beta$ and GDF11. These secretions would take place to short and shift the ADSCs profile to a less inflammatory profile and enhance angiogenesis, cell migration and proliferation.

\section{Conclusion}

The capacity of ADSCs to proliferate and self-renew in vitro as well as in vitro, added to their innate differentiation targeted more scientific advancements in the field of regenerative medicine. In normal conditions and through their enriched secretome, these cells orchestrate the mechanisms related to cell differentiation, proliferation and cell migration even by upregulating genes involved in skin barrier function, in cellular proliferation and epidermal turnover and differentiation. Nevertheless, these regulations appear to be attained by their immunomodulating ability through their paracrine and autocrine pathways as well as cell-to-cell contact.

In wound defects, ADSCs migrate rapidly towards the wounded sites where process of cell differentiation into skin cell components occurs. However, ADSCs participate more likely to all the phases of wound healing through autocrine and paracrine pathways. Otherwise, during aging senescent cells increased and the paracrine senescent secretome of ADSCs can trigger and reinforce inflammation within their microenvironment [55]. Macrophages are considered the most immune cells involved in the innate skin immune surveillance, but recently as the key regulator of skin immunomodulation as they infiltrate skin layers in inflammation-associated diseases and during aging.
Interactions between resident skin ADSCs and local macrophages lead to changes in the immunological phenotype of both cells which is reflected by the differentiation of M2 anti-inflammatory profile, the suppression of Th cells and the increase in Treg cell activation [74]. ADSC's changes are also relevant through the secretion of anti-inflammatory cytokines and the increase in the expression of genes involved in skin homeostasis [29]. Adding to their direct skin cell contact, these cells orchestrate and accelerate the mechanisms supporting their differentiation into DF and keratinocytes, melanin production, neo-angiogenesis and increase in capillary density and finally the reepithelialization and wound closure [32]. The reciprocal interactions between ADSCs and macrophages let us to suggest that dermal composition in ADSCs and macrophages is the key regulator of the skin immune profile $[32,75]$. Their respective number would impact the inflammatory status of the skin and consequently skin cell regeneration and function in both normal and wounded conditions by modulating the fate of ADSCs [74]. Indeed, a recent work has confirmed that the immunomodulatory effect of ADSCs is significantly dependent on their dose in an animal model [76].

Moreover, the clinical efficacy of ADSCs remains dependent of the number of cells injected their route of administration and their location's origin as well. Visceral ADSCs secrete higher quantities of inflammatory cytokines IL6 , IL-8 and TNF- $\alpha$ when compared to the subcutaneous one's [72].

Other point of view to consider is that patient-associated factors lead to inherent variability relative to ADSCs viability, self-renewal ability, differentiation potency and their secretory profile containing immunomodulatory mediators. Collected from donors presenting medical histories such as breast cancer, Croh'n diseases or other inflammatory diseases, ADSCs present an inflammation induced secretome associated to their self-renewal capacity $[68,77]$. This suggested that the in vitro or in vivo preconditioning of ADSCs could open the way to potential new strategies for treating skin inflammations.

This might be useful and should be considered when designing new clinical therapies using ADSCs or their specific exosomes whereby their immunomodulation activities occur.

Acknowledgment: The authors wish to thank the University of Mohammed VI Polytechnic for funding this work.

Authors Contributions: All authors participated in the research and writing of this manuscript.

Conflicts of Interest: The authors declare no conflict of 


\section{Journal of Embryology \& Stem Cell Research}

interest.

\section{References}

1. Marfia G, Navone SE, Di Vito C, Ughi N, Tabano S, et al. (2015) Mesenchymal stem cells: potential for therapy and treatment of chronic non-healing skin wounds. Organogenesis 11(4): 183-206.

2. Zhang Y, Wei Y, Liu D, Liu F, Li X, et al. (2017) Role of growth differentiation factor 11 in development, physiology and disease. Oncotarget 8(46): 81604-81616.

3. Hyldig K, Riis S, Pennisi CP, Zachar V, Fink T (2017) Implications of Extracellular Matrix Production by Adipose Tissue-Derived Stem Cells for Development of Wound Healing Therapies. Int J Mol Sci 18(6).

4. Sheykhhasan M, Wong JKL, Seifalian AM (2019) Human Adipose-Derived Stem Cells with Great Therapeutic Potential. Curr Stem Cell Res Ther 14(7): 532-548.

5. Bacakova L, Zarubova J, Travnickova M, Musilkova J, Pajorova, et al. (2018) Stem cells: their source, potency and use in regenerative therapies with focus on adiposederived stem cells: a review. Bio technol Adv 36(4): 1111-1126.

6. Kilroy GE, Foster SJ, Wu X, Ruiz J, Sherwood S, et al. (2007) Cytokine profile of human adipose- derived stem cells: expression of angiogenic, hematopoietic, and proinflammatory factors. J Cell Physiol 212(3): 702-709.

7. Park BS, Jang KA, Sung JH, Park JS, Kwon YH, et al. (2008) Adipose-derived stem cells and their secretory factors as a promising therapy for skin aging. Dermatol Surg 34(10): 1323-1326.

8. Balaji S, Keswani SG, Crombleholme TM (2012) The Role of Mesenchymal Stem Cells in the Regenerative Wound Healing Phenotype. Adv Wound Care (New Rochelle) 1(4): 159-165.

9. Amable PR, Teixeira MVT, Carias RBV, Granjeiro JM, Borojevic R (2014) Protein synthesis and secretion in human mesenchymal cells derived from bone marrow, adipose tissue and Wharton's jelly. Stem Cell Res Ther 5(2): 53.

10. Elman JS, Murray RC, Murray RM, Wang F, Shen K, et al. (2014) Pharmacokinetics of natural and engineered secreted factors delivered by mesenchymal stromal cells. PLoS ONE 9: e89882.

11. Choi EW, Seo MK, Woo EY, Kim SH, Park EJ, et al. (2018) Exosomes from human adipose-derived stem cells promote proliferation and migration of skin fibroblasts. Exp Dermatol 27(10): 1170-1172.

12. An Y,Zhao J, Nie F, Qin Z, Xue H, etal. (2019) Exosomes from Adipose-Derived Stem Cells (ADSCs) Overexpressing miR-21 Promote Vascularization of Endothelial Cells. Sci Rep 9: 12861.

13. Harada M, Jinnin $M$, Wang Z, Hirano A, Tomizawa $Y$ (2017) The expression of miR-124 increases in aged skin to cause cell senescence and it decreases in squamous cell carcinoma. Biosci Trends 10(6): 454-459.

14. Waters R, Subham S, Pacelli S, Modaresi S, Chakravarti AR, et al. (2019) Development of MicroRNA-146a-Enriched Stem Cell Secretome for Wound-Healing Applications. Mol Pharm 16(10): 4302-4312.

15. Gonzalez Rey E, Anderson P, Gonzalez MA, Rico L, Buscher D, et al. (2009) Human adult stem cells derived from adipose tissue protect against experimental colitis and sepsis. Gut 58(7): 929-939.

16. Koh KS, Oh TS, Kim H, Chung IW, Lee KW, et al. (2012) Clinical application of human adipose tissue-derived mesenchymal stem cells in progressive hemifacial atrophy (Parry-Romberg disease) with microfat grafting techniques using 3-dimensional computed tomography and 3- dimensional camera. Ann Plast Surg 69(3): 331337.

17. Bae YC, Song JS, Bae SH, Kim JH (2015) Effects of human adipose-derived stem cells and stromal vascular fraction on cryopreserved fat transfer. Dermatol Surg 41(5): 605614.

18. Eto H, Kato H, Suga H, Aoi N, Doi K, et al. (2012) The fate of adipocytes after nonvascularized fat grafting: evidence of early death and replacement of adipocytes. Plast Reconstr Surg 129(5): 1081-1092.

19. Ferreira A, Da F, Gomes DA (2018) Stem Cell Extracellular Vesicles in Skin Repair. Bioengineering (Basel) 6(1).

20. Kim JH, Jung M, Kim HS, Kim YM, Choi EH (2011) Adiposederived stem cells as a new therapeutic modality for ageing skin. Exp Dermatol 20(5): 383-387.

21. Zarei F, Abbaszadeh A (2018) Stem cell and skin rejuvenation. Journal of Cosmetic and Laser Therapy 20(3): 193-197.

22. Sheng L, Yang M, Liang Y, Li Q (2013) Adipose tissuederived stem cells (ADSCs) transplantation promotes regeneration of expanded skin using a tissue expansion model. Wound Repair Regen 21(5): 746-754. 


\section{Journal of Embryology \& Stem Cell Research}

23. Collawn SS, Banerjee NS, De la Torre J, Vasconez L, Chow LT (2012) Adipose-derived stromal cells accelerate wound healing in an organotypic raft culture model. Ann Plast Surg 68(5): 501-504.

24. Xia S, Zhang X, Zheng S, Khanabdali R, Kalionis B, et al. (2016) An Update on Inflamm-Aging: Mechanisms, Prevention, and Treatment. J Immunol Res 2016: 8426874 .

25. Schafer MJ, Atkinson EJ, Vanderboom PM, Kotajarvi B, White TA, et al. (2016) Quantification of GDF11 and Myostatin in Human Aging and Cardiovascular Disease. Cell Metab 23(6): 1207-1215.

26. Glass DJ (2016) Elevated GDF11 Is a Risk Factor for AgeRelated Frailty and Disease in Humans. Cell Metab 24(1): 7-8.

27. Othmani AE, Rouam S, Abbad A, Erraoui C, Harriba S, et al. (2019) Cryopreservation Impacts Cell Functionality of Long Term Expanded Adipose-Derived Stem Cells. Journal of Stem Cell Research \& Therapy 9: 1.

28. Kim YJ, Seo DH, Lee SH, Lee SH, An GH, et al. (2018) Conditioned media from human umbilical cord bloodderived mesenchymal stem cells stimulate rejuvenation function in human skin. Biochem Biophys Rep 16: 96102.

29. Idkowiak Baldys J, Santhanam U, Buchanan SM, Pfaff KL, Rubin LL, et al. (2019) Growth differentiation factor 11 (GDF11) has pronounced effects on skin biology. PLoS ONE 14(6): e0218035.

30. Bellei B, Migliano E, Tedesco M, Caputo S, Papaccio F, et al. (2018) Adipose tissue-derived extracellular fraction characterization: biological and clinical considerations in regenerative medicine. Stem Cell Res Ther 9(1): 207.

31. Hong SJ, Jia SX, Xie P, Xu W, Leung KP, et al. (2013) Topically delivered adipose derived stem cells show an activated-fibroblast phenotype and enhance granulation tissue formation in skin wounds. PLoS ONE 8(1): e55640.

32. Mazini L, Rochette L, Admou B, Amal S, Malka G (2020) Hopes and limits of Adipose-Derived Stem Cells (ADSCs) and Mesenchymal Stem Cells (MSCs) in Wound Healing. Int J Mol Sci 21.

33. Blanpain C, Fuchs E (2009) Epidermal homeostasis: a balancing act of stem cells in the skin. Nat Rev Mol Cell Biol 10(3): 207-217.

34. Blanpain C, Horsley V, Fuchs E (2007) Epithelial stem cells: turning over new leaves. Cell 128(3): 445-458.
35. Blanpain C, Fuchs E (2006) Epidermal stem cells of the skin. Annu Rev Cell Dev Biol 22: 339-373.

36. Monfort A, Soriano Navarro M, Garcia Verdugo JM, Izeta A (2013) Production of human tissue-engineered skin trilayer on a plasma-based hypodermis. J Tissue Eng Regen Med 7(6): 479-490.

37. Stojanovic S, Najman S (2019) The Effect of Conditioned Media of Stem Cells Derived from Lipoma and Adipose Tissue on Macrophages' Response and Wound Healing in Indirect Co-culture System In Vitro. Int J Mol Sci 20(7).

38. Salvioli S, Capri M, Valensin S, Tieri P, Monti D, et al. (2006) Inflamm-aging, cytokines and aging: state of the art, new hypotheses on the role of mitochondria and new perspectives from systems biology. Curr Pharm Des 12(24): 3161-3171.

39. Nurkovic J, Dolicanin Z, Mustafic F, Mujanovic R, Memic $M$, et al. (2016) Mesenchymal stem cells in regenerative rehabilitation. J Phys Ther Sci 28(6): 1943-1948.

40. Barrientos S, Stojadinovic O, Golinko MS, Brem H, Tomic Canic M (2008) Growth factors and cytokines in wound healing. Wound Repair Regen 16(5): 585-601.

41. Ferreira A, Da F, Cunha P, Da S, Carregal VM, et al. (2017) Extracellular Vesicles from Adipose-Derived Mesenchymal Stem/Stromal Cells Accelerate Migration and Activate AKT Pathway in Human Keratinocytes and Fibroblasts Independently of miR-205 Activity. Stem Cells Int 2017: 14.

42. Rehman J, Traktuev D, Li J, Merfeld Clauss S, Temm Grove CJ, et al. (2004) Secretion of angiogenic and antiapoptotic factors by human adipose stromal cells. Circulation 109(10): 1292-1298.

43. Benard PV, Silvestre JS, Cousin B, Andre M, Nibbelink M, et al. (2004) Plasticity of human adipose lineage cells toward endothelial cells: physiological and therapeutic perspectives. Circulation 109(5): 656-663.

44. Hur W, Lee HY, Min HS, Wufuer M, Lee CW, et al. (2017) Regeneration of full-thickness skin defects by differentiated adipose-derived stem cells into fibroblastlike cells by fibroblast-conditioned medium. Stem Cell Res Ther 8(1): 92.

45. Komaki M, Numata Y, Morioka C, Honda I, Tooi M, et al. (2017) Exosomes of human placenta- derived mesenchymal stem cells stimulate angiogenesis. Stem Cell Res Ther 8(1): 219.

46. Bachmann S, Jennewein M, Bubel M, Guthorl S, Pohlemann T, et al. (2019) Interacting adipose-derived 


\section{Journal of Embryology \& Stem Cell Research}

stem cells and microvascular endothelial cells provide a beneficial milieu for soft tissue healing. Mol Biol Rep 47(1): 111-122.

47. Lynch SE, Nixon JC, Colvin RB, Antoniades HN (1987) Role of platelet- derived growth factor in wound healing: synergistic effects with other growth factors. Proc Natl Acad Sci 84(21): 7696-7700.

48. Pierce GF, Mustoe TA, Lingelbach J, Masakowski VR, Griffin GL, et al. (1989) Platelet-derived growth factor and transforming growth factor-beta enhance tissue repair activities by unique mechanisms. J Cell Biol 109(1): 429-440.

49. Ucuzian AA, Gassman AA, East AT, Greisler HP (2010) Molecular mediators of angiogenesis. J Burn Care Res 31(1): 158-175.

50. Lozito TP, Jackson WM, Nesti LJ, Tuan RS (2014) Human mesenchymal stem cells generate a distinct pericellular zone of MMP activities via binding of MMPs and secretion of high levels of TIMPs. Matrix Biol 34: 132-143.

51. Xiao W, Tang H, Wu M, Liao Y, Li K, et al. (2017) Ozone oil promotes wound healing by increasing the migration of fibroblasts via PI3K/Akt/mTOR signaling pathway. Biosci Rep 37(6).

52. Koivisto L, Bi J, Hakkinen L, Larjava H (2018) Integrin $\alpha v \beta 6$ : Structure, function and role in health and disease. Int J Biochem Cell Biol 99: 186-196.

53. Sumi M, Sata M, Toya N, Yanaga K, Ohki T, et al. (2007) Transplantation of adipose stromal cells, but not mature adipocytes, augments ischemia-induced angiogenesis. Life Sci 80(6): 559-565.

54. Liu Y, Cox SR, Morita T, Kourembanas S (1995) Hypoxia regulates vascular endothelial growth factor gene expression in endothelial cells. Identification of a 5' enhancer. Circ Res 77(3): 638-643.

55. Lunyak VV, Amaro Ortiz A, Gaur M (2017) Mesenchymal Stem Cells Secretory Responses: Senescence Messaging Secretome and Immunomodulation Perspective. Front Genet 8: 220.

56. Seppanen E, Roy E, Ellis R, Bou Gharios G, Fisk NM, et al. (2013) Distant mesenchymal progenitors contribute to skin wound healing and produce collagen: evidence from a murine fetal microchimerism model. PLoS ONE 8: e62662.

57. Waterman RS, Tomchuck SL, Henkle SL, Betancourt AM (2010) A new mesenchymal stem cell (MSC) paradigm: polarization into a pro-inflammatory MSC1 or an
Immunosuppressive MSC2 phenotype. PLoS ONE 5: e10088.

58. Park JE, Barbul A (2004) Understanding the role of immune regulation in wound healing. Am J Surg 187(5A): 11S-16S.

59. Anderson PH (2017) Vitamin D Activity and Metabolism in Bone. Curr Osteoporos Rep 15(5): 443-449.

60. Baharlou R, Ahmadi Vasmehjani A, Faraji F, Atashzar MR, Khoubyari M, et al. (2017) Human adipose tissuederived mesenchymal stem cells in rheumatoid arthritis: Regulatory effects on peripheral blood mononuclear cells activation. Int Immunopharmacol 47: 59-69.

61. Domenis R, Cifu A, Quaglia S, Pistis C, Moretti M, et al. (2018) Pro inflammatory stimuli enhance the immunosuppressive functions of adipose mesenchymal stem cells-derived exosomes. Sci Rep 8(1): 13325.

62. Kronsteiner B, Wolbank S, Peterbauer A, Hackl C, Redl $\mathrm{H}$, et al. (2011) Human mesenchymal stem cells from adipose tissue and amnion influence T-cells depending on stimulation method and presence of other immune cells. Stem Cells Dev 20(12): 2115-2126.

63. Li W, Wang W, Liu L, Qu R, Chen X, et al. (2019) GDF11 antagonizes TNF- $\alpha$-induced inflammation and protects against the development of inflammatory arthritis in mice. FASEB J 33(3): 3317-3329.

64. Li T, Ma H, Ma Z, Qiang L, Yang Z, et al. (2019) Mussel-Inspired Nanostructures Potentiate the Immunomodulatory Properties and Angiogenesis of Mesenchymal Stem Cells. ACS Appl Mater Interfaces 11(19): 17134-17146.

65. Liang J, Zhang H, Kong W, Deng W, Wang D, et al. (2018) Safety analysis in patients with autoimmune disease receiving allogeneic mesenchymal stem cells infusion: a long-term retrospective study. Stem Cell Res Ther 9(1): 312.

66. Klar AS, Michalak Mićka K, Biedermann T, Simmen Meuli C, Reichmann E, et al. (2018) Characterization of M1 and M2 polarization of macrophages in vascularized human dermo-epidermal skin substitutes in vivo. Pediatr Surg Int 34(2): 129-135.

67. Qin S, Zheng JH, Xia ZH, Qian J, Deng CL, et al. (2019) CTHRC1 promotes wound repair by increasing M2 macrophages via regulating the TGF- $\beta$ and notch pathways. Biomed Pharmacother 113: 108594.

68. Wang AS, Dreesen O (2018) Biomarkers of Cellular Senescence and Skin Aging. Front Genet 9: 247. 


\section{Journal of Embryology \& Stem Cell Research}

69. Revu S, Wu J, Henkel M, Rittenhouse N, Menk A, et al. (2018) IL-23 and IL-1 $\beta$ Drive Human Th17 Cell Differentiation and Metabolic Reprogramming in Absence of CD28 Costimulation. Cell Rep 22(10): 26422653.

70. Mei W, Xiang G, Li Y, Li H, Xiang L, et al. (2016) GDF11 Protects against Endothelial Injury and Reduces Atherosclerotic Lesion Formation in Apolipoprotein E-Null Mice. Mol Ther 24(11): 1926-1938.

71. Das T, Chen Z, Hendriks RW, Kool M (2018) A20/ Tumor Necrosis Factor $\alpha$ - Induced Protein 3 in Immune Cells Controls Development of Autoinflammation and Autoimmunity: Lessons from Mouse Models. Front Immunol 9: 104.

72. Ritter A, Friemel A, Roth S, Kreis NN, Hoock SC, et al. (2019) Subcutaneous and Visceral Adipose- Derived Mesenchymal Stem Cells: Commonality and Diversity. Cells 8(10): 1288.

73. RodriguezLA, MohammadipoorA,AlvaradoL, Kamucheka RM, Asher AM, et al. (2019) Preconditioning in an Inflammatory Milieu Augments the Immunotherapeutic Function of Mesenchymal Stromal Cells. Cells 8(5): 462.
74. Ozdemir RBO, Ozdemir AT, Sarıboyacı AE, Uysal O, Tuglu MI, et al. (2019)The investigation of immunomodulatory effects of adipose tissue mesenchymal stem cell educated macrophages on the CD4 T cells. Immunobiology 224(4): 585-594.

75. Bobyleva P, Gornostaeva A, Andreeva E, Ezdakova M, Gogiya B, et al. (2019) Reciprocal modulation of cell functions upon direct interaction of adipose mesenchymal stromal and activated immune cells. Cell Biochem Funct 37(4): 228-238.

76. Lee OJ, Luk F, Korevaar SS, Koch TG, Baan CC, et al. (2020) The Importance of Dosing, Timing, and (in)Activation of Adipose Tissue-Derived Mesenchymal Stromal Cells on Their Immunomodulatory Effects. Stem Cells Dev 29(1): 38-48.

77. Parsons AM, Ciombor DM, Liu PY, Darling EM (2018) Regenerative Potential and Inflammation-Induced Secretion Profile of Human Adipose-Derived Stromal Vascular Cells Are Influenced by Donor Variability and Prior Breast Cancer Diagnosis. Stem Cell Rev Rep 14(4): 546-557. 